\title{
Rasgos de identidad. Tres perspectivas epistemológicas de la contabilidad social y ambiental
}

\section{Héctor José Sarmiento}

Programa de Contaduría Pública

Politécnico Colombiano Jaime Isaza Cadavid

Este trabajo busca rasgos de identidad epistémica, y explora la existencia de una comunidad científica en el campo de la contabilidad social y ambiental. Para ello, conecta elementos epistemológicos, como la clasificación habermasiana de las ciencias, con los enfoques de investigación de la teoría crítica a partir del análisis de una selección de textos paradigmáticos de dicho campo de conocimiento. Se hace un análisis de contenido documental guiado por 3 categorías y 48 subcategorías de análisis de texto, que permiten vincular algunos textos/autores con una perspectiva epistemológica/ metodológica. Este ejercicio logra agrupar los textos en torno a algunos rasgos epistemológicos, pero la ubicación de los autores no es factible de manera categórica por razones que se explicitan. Se reconocen algunas limitaciones metodológicas en torno al alcance y la validación que abren algunas líneas de trabajo hacia el futuro.

Palabras clave: contabilidad social y ambiental, teoría contable, contabilidad de sostenibilidad, heterodoxia contable

\section{Identity Traits.Three Epistemological Perspectives of Social and Environmental Accounting}

This work looks for traits of epistemic identity and explores the existence of a scientific community in the field of social and environmental accounting. To do this, it connects epistemological elements such as the Habermasian classification of the sciences with the research approaches of critical theory, in the analysis of a selection of paradigmatic texts from that field of knowledge. An analysis of documentary content, guided by three categories and 48 subcategories of text analysis is made, which allows linking some text/authors with an epistemological/methodological perspective. This exercise manages to group the texts around some epistemological features, but the categorical location of the authors is not feasible for reasons that are made explicit there. Some methodological limitations regarding scope and validation are recognized, which open some lines of work for the future.

Keywords: social and environmental accounting, accounting theory, sustainability accounting, accounting heterodoxy

\section{Traços de Identidade. Três perspectivas epistemológicas da contabilidade social e ambiental}

Este trabalho procura traços de identidade epistêmica e explora a existência de uma comunidade científica no campo da contabilidade social e ambiental. Para isso, conecta elementos epistemológicos, como a classificação Habermasiana das ciências, com as abordagens de pesquisa da teoria crítica, na análise de uma seleção de textos paradigmáticos desse campo do conhecimento. É feita uma análise do conteúdo documental, guiada por três categorias e 48 subcategorias de análise de texto, o que permite vincular alguns textos / autores a uma perspectiva epistemológica / metodológica. Este exercício consegue agrupar os textos em torno de algumas características epistemológicas, mas a localização categórica dos autores não é viável por razôes explicitadas ali. São reconhecidas algumas limitações metodológicas em relação ao escopo e validação, que abrem algumas linhas de trabalho para o futuro.

Palavras-chave: contabilidade social e ambiental, teoria contábil, contabilidade de sustentabilidade, heterodoxia contábil 


\section{Introducción}

La concepción de la contabilidad como una disciplina de conocimiento influida por factores del contexto social ha permitido la consolidación de una vertiente de pensamiento contable heterodoxo cada vez más amplia, inclusiva e influyente. Esta se conecta con las problemáticas sociales y ambientales (Gray, Owen \& Adams, 1996) a partir de los desarrollos en medición, valoración, interpretación, evaluación, y control de los hechos y fenómenos del contexto. Desde la perspectiva teórica tradicional $\mathrm{u}$ ortodoxa (mainstream), y bajo el amparo del paradigma de la utilidad de la información, la contabilidad actúa como un sistema de información, una institución o una disciplina tecnológica (Wahyudi, 1999) que se ocupa del procesamiento sistemático de información útil para la toma de decisiones. Entre las decisiones a considerar, hoy se privilegian aquellas referidas a la inversión en los mercados de valores (Khurana \& Raman, 2004). En esa dirección, los objetivos de la contabilidad están orientados a propiciar las condiciones que les permitan a los usuarios reducir la incertidumbre en escenarios de decisión mediante la preparación de información pertinente para la toma de decisiones de inversión en ambientes con altos niveles de riesgo financiero y similares oportunidades de rentabilidad.

En oposición a los ideales, fines y medios de la mainstream, la corriente heterodoxa, privilegia valores como la justicia social, el compromiso ambiental y la transparencia informativa. Estos factores demandarían una revisión de la disciplina en términos de conceptos, metodologías e instrumentos de trabajo (Tinker \& Gray, 2003). No obstante, no se ha trabajado suficiente en construir una estructura teórica que sustente los desarrollos de la relación contabilidadambiente, aspecto que podría ser necesario o al menos deseable para dotar de mayor solidez conceptual a el área disciplinar. Llama la atención que numerosas metodologías, técnicas, e instrumentos de medición, valoración, control, y análisis de la contabilidad social y ambiental (en adelante, "CSA») han surgido a partir de conceptos y teorías que privilegian objetivos de acumulación de capital financiero (Thomson, 2015). A pesar de ser muy significativos e importantes, la mayoría de los avances en el campo de CSA no cuestionan los fundamentos teóricos de la contabilidad (Gaffikin, 2006). Incluso, algunos autores asumen los fundamentos teóricos de la contabilidad financiera (Burrit \& Schaltegger, 2001; Solomon \& Solomon, 2006; Guidry \& Patten, 2012) para hablar de CSA, como si no hubiese diferencias entre dichos campos de conocimiento.

Desde la perspectiva heterodoxa, la CSA debería contribuir a tomar decisiones de producción, distribución, y redistribución de la riqueza material, social y natural en consonancia con la sustentabilidad de las fuentes que la hacen posible (Ariza, León \& Gómez, 2006). Sin embargo, los espacios académicos y profesionales de la contabilidad están dominados por los intereses económicos y políticos del capitalismo financiero internacional (Tinker, Neimark \& Lehman, 1991; Archel, Husillos \& Spence, 2011). Tales intereses muestran un impacto distinto en los productores y usuarios de información contable al posicionarlos en los extremos del escenario conceptual. Desde sus respectivas visiones, las ciencias sociales se han ocupado de estudiar los fenómenos que rodean esta dinámica del desarrollo. Particularmente, la CSA ha centrado su interés en las múltiples desigualdades de información que determinan las inequidades propias del capitalismo financiero, sus efectos sobre el conjunto de la sociedad y los ecosistemas, y sus respectivas interacciones (Puxty, Willmott, Cooper \& Lowe, 1987; Gómez, 2006; Martínez \& Gómez-Villegas, 2015; Gil, 2018). 
En el punto medio entre la corriente ortodoxa y la heterodoxa de la contabilidad, este artículo intenta conectar la categorización de las ciencias según la escuela de Frankfurt (Habermas, 1995) con los enfoques metodológicos de investigación de Burrell y Morgan (1979) para delinear algunos perfiles epistemológicos de la CSA. Tal conexión se construye a través del examen no parametral de algunos textos representativos de la CSA que son examinados mediante la técnica de análisis de contenido. Esto permite identificar qué características conceptuales (categorías) distinguen el trabajo en cada uno de los enfoques epistemológicos y qué matices existen dentro de los mismos. El artículo intenta hacer una contribución a partir de la conexión de dos desarrollos epistemológicos de alto prestigio académico para identificar enfoques en la literatura contable de CSA que faciliten el estudio sistemático de este campo de conocimiento en los procesos de investigación y, eventualmente, en la identificación de una comunidad científica en este campo.

\section{Metodología}

La investigación que precede este trabajo se fundamenta en un ejercicio de análisis de literatura orientado a identificar elementos conceptuales que permitan hacer una clasificación epistemológica de un campo disciplinar. Para lograrlo, se parte de una revisión no parametral de literatura (Zemelman, 2004), que contempla 3 categorías de análisis con 48 factores de búsqueda y análisis de contenido con el propósito de rastrear conexiones entre enfoques y referentes conceptuales (Aktouf, 2011; Fassio, 2018) que permitan la caracterización epistemológica deseada.
Se han analizado 78 artículos de 35 autores $^{1}$, este no es un dato determinante, pues la validación se centra en las posturas, expresiones y razones de los autores con respecto a los problemas de la CSA. Los textos/ autores considerados son Carol Adams, Pablo Archel, Amanda Ball, Jan Bebbington, Ahmed Belkaoui, Jane Broadbent, Roger Burrit, Carmen Correa, Charles Cho, Craig Deegan, Jesse Dillard, Marc Epstein, Michael Gaffikin, Rob Gray, James Guthrie, Anthony Hopwood, Javier Husillos, Matias Laine, Carlos Larrinaga, Cheryl Lehman, Tony Lowe, Reg Mathews, José Mariano Moneva, Brendan O'Dwyer, David Owen, Lee Parker, John Peloza, Lee Preston, Anthony Puxty, Yan Qiu, Aris Solomon, Crawford Spence, Tony Tinker, Ian Thomson y Jeffrey Unerman. Algunos trabajos analizados fueron escritos en coautoría entre los autores de esta selección o con otros autores no considerados en la lista.

La selección de los textos es una muestra intencionada y guiada por su citación reiterada en la literatura previa y el libre acceso a los artículos examinados. Se rastrean ideas, conceptos y afirmaciones que se conectan con los enfoques epistemológicos y metodológicos (Aktouf, 2011) que se describen más adelante en las consideraciones teóricas. Este diseño cualitativo no pretende hacer categorizaciones o clasificaciones bajo criterios de representatividad, precisión o exactitud (Galeano, 2004), sino identificar atributos vinculantes entre la teoría epistemológica y los textos de CSA, y entre estos últimos, para visualizar los matices epistémicos del campo disciplinal de la CSA, lo cual ayudará a los nuevos investigadores a reconocer tendencias de pensamiento en esta área y, de forma indirecta, a los autores vinculados con ellas.

1 Códigos de autores: ADM, ARC, BAL, BBT, BLK, BDT, BRT, COR, CHO, DEG, DLR, EPS, GFK, GRY, GTR, HPW, HUS, LAN, LRG, LHM, LOW, MTW, MNV, ODW, OWN, PKR, PLZ, PST, PXT, QIU, SOL, SPN, TNK, TMS, UNM. 
Para hacer el trabajo previsto, se procedió a seleccionar algunos textos que se consideran seminales (libros, capítulos) y artículos arquetípicos que provienen de algunas revistas que, aunque tienen perspectivas diferentes, son muy representativas en el campo de la CSA: Accounting, Organizations and Society, Critical Perspectives on Accounting, Accounting, Auditing o Accountability Journal, Accounting Forum, Business, Strategy and the Environment, y The British Accounting Review, entre otras. La búsqueda de los elementos conceptuales se hizo sobre la base de las características de algunos enfoques epistemológicos de las ciencias sociales (Habermas, 1988; Gadamer, 1992; Foucault, 1987), que luego han sido aplicados a las ciencias económicas y, posteriormente, a la contabilidad (Burrell \& Morgan, 1979). Esto permite observar cambios en las posiciones de los autores a través del tiempo, por ello, los textos analizados corresponden a diferentes etapas de su vida académica.

\section{Algunas consideraciones teóricas}

Desde que se hizo inevitable la brecha metodológica entre las ciencias naturales y las ciencias sociales, estas últimas también entraron en un proceso de especialización y subdivisión que ha determinado la aparición de diversos enfoques de descripción y explicación científica para el desarrollo del conocimiento. De forma casi paralela al gran debate epistemológico del siglo XX que sostuvieron Popper (2008), Kuhn (2006), Lákatos (1998), Laudan (1986) y Feyerabend (2003), se fue desarrollando una discusión epistemológica en el campo de las ciencias sociales, inicialmente impulsada por los filósofos de la escuela de Frankfurt. El debate supuso la distinción conceptual de las ciencias sociales y las ciencias humanas, lo que ha dejado como resultado una amplia oferta de posibilidades ontológicas y metodológicas para el desarrollo de la investigación en cada campo.
Dentro de las propuestas metodológicas para el estudio de las disciplinas, merece especial mención la clasificación propuesta por Habermas en Conocimiento e interés (1995). El filósofo señala que, más allá de los objetos de estudio y los métodos para abordarlos, es mejor y más importante considerar el papel del trabajo, el lenguaje y la interacción social en la construcción de las ciencias sociales en la contemporaneidad. A partir de la forma como cada uno de estos tres elementos incide y predomina en los procesos de investigación científica, emerge una clasificación que identifica como «categorías taxonómicas»: las ciencias empíricoanalíticas, adheridas a la idea de trabajo y herederas de la tradición epistemológica de las ciencias naturales; las ciencias histórico-hermenéuticas, dominadas por el lenguaje y los procesos de la interpretación; y las ciencias crítico-sociales, inmersas en el estudio de la intersubjetividad propia de la interacción de los colectivos humanos (Habermas, 1995; Vasco, 1990).

En concordancia con esta perspectiva epistemológica, las ciencias sociales económicas han incorporado clasificaciones particulares para sus propios desarrollos. Esto permite reconocer posiciones tradicionales (ortodoxia) y radicales (heterodoxia) dentro de una misma disciplina o campo de conocimiento científico (Burrell \& Morgan, 1979; Chua, 1986). De esta manera, han surgido grandes debates aún en curso que enfrentan perspectivas casi siempre opuestas, que corresponden por reflejo al legado de autores, y escuelas de pensamiento económico y administrativo antagónicas, entre las oposiciones vigentes, se encuentran la de la economía financiera, representada por autores como Friedmann, Megginson o Petras; la economía social, liderada por Stiglitz, Sen, o Max Neff; la administración estratégica, con nombres como Porter, Thompson o Kaplan; y la escuela humanista de la gestión, en la que destacan De Gaulejac, Aktouf, Chanlat y Bédard (Aktouf, 2010). 
En ese camino, la contabilidad empezó un proceso de especialización. Así como este ha enriquecido, ampliado y profundizado el acervo de conocimiento, también ha abierto importantes brechas entre los académicos y profesionales que defienden la ortodoxia financiera de la contabilidad, heredera de la tradición economicista; y quienes abogan por los desarrollos alternativos de la disciplina (Dillard, 1991; Gracia, 2013), derivados de la concepción sociopolítica de las ciencias sociales. Así, el desarrollo de la investigación en CSA ha evolucionado a partir de considerar que la contabilidad financiera no ofrece el espacio, ni las metodologías y técnicas necesarias y adecuadas para describir, analizar, interpretar y reportar información referida a la influencia de la actividad económica de las organizaciones sobre el ambiente (Ullmann, 1976; Dierkes \& Preston, 1977; Owen, Swift, Bowerman \& Humphrey, 2000; Gray \& Bebbington, 2001). Esto ha hecho necesario que se abran diferentes campos de trabajo que implican consecuentes compromisos (Adams $\&$ Larrinaga, 2007) en al menos tres direcciones:

a) un primer enfoque, más técnico y cercano a la tradición contable financiera, interesado en proponer mecanismos de medición, valoración y control de las relaciones empresa-ambiente, orientado a legitimar la actividad empresarial en función de sus objetivos económicos;

b) un enfoque alternativo, dirigido a describir e interpretar la información y los intereses que subyacen en las relaciones empresa-ambiente como parte de la realidad socioeconómica del mundo contemporáneo, sujeto a múltiples interacciones derivadas de la complejidad de los procesos de desarrollo; y

c) un tercer enfoque más radical, interesado en poner en conflicto los dos enfoques anteriores y a cada uno consigo mismo, en atención a la necesidad de que la contabilidad se comprometa con las transformaciones que exige el estado de crisis en que se encuentra el actual modelo de desarrollo de Occidente (Burrell \& Morgan, 1979; Larrinaga, 2017).

La yuxtaposición semántica y semiótica de las posiciones gnoseológicas y epistemológicas, producto también de los usos idiomáticos, representan una importante tensión para muchas disciplinas y, por consiguiente, para el campo disciplinal de la CSA. Aunque algunos autores de este campo asimilan gnoseología como epistemología (Gaffikin, 2006; Dillard, 2014), puede entenderse que la epistemología actúa como una filosofía de la ciencia y se ocupa de estudiar las formas en que una teoría llega a ser considerada como científica. Asimismo, la epistemología estudia qué reglas deben cumplirse para que la teoría sea científica, qué factores determinan su crecimiento y consolidación, y qué condiciones determinan que deje de ser ciencia (debate Popper-Lákatos-Kuhn). A su vez, esto permite ubicar una teoría y a su autor en una determinada escuela de pensamiento dentro de una ciencia o disciplina en particular; y, por oposición, a sus contradictores en una escuela distinta o incluso adversaria. Naturalmente, estos procesos tienen implicaciones y compromisos gnoseológicos en función del método utilizado para la construcción teórica, pero debe haber claridad en que una epistemología de la contabilidad solo es posible si se asume esta disciplina como un cuerpo de conocimiento con poder constituyente en el terreno de lo científico (Dillard, 1991; Lopes de Sá, 1995; Lodh \& Gaffikin, 1997). Esto se aplica también para el campo disciplinal de la CSA (Carrasco \& Larrinaga, 1996). Por contraste, habrá que decir que es conceptualmente imposible una epistemología de la contabilidad si esta se asume como un sistema de información, una técnica o cualquier otra noción que no corresponda con una categoría de conocimiento científico. Tam- 
bién habrá que distinguir la esencia de la contabilidad (dimensión ontológica) en tanto saber disciplinar, de las formas de uso (dimensión metodológica) que de ella se haga en un contexto determinado, pues esto es lo que posibilita que se utilice como una tecnología de racionalización, de acuerdo a lo propuesto por Weber (2003); o incluso como una tecnología de dominación, según afirma Foucault (1987).

Partiendo de esa idea y asumiendo que la CSA es un campo disciplinal de la contabilidad, podrán examinarse, desde una perspectiva epistemológica, las teorías, proposiciones y conceptos con los cuales se describen y explican las relaciones entre el saber contable, las organizaciones, el ambiente y el conjunto de la sociedad. Así, el avance cualitativo de las ciencias sociales también tocó las puertas de la contabilidad, por lo que la concepción utilitarista de la disciplina fue puesta en tela de juicio. Esto favoreció la aparición de nuevas concepciones que ponen a la contabilidad y la investigación contable en contacto con las personas, las comunidades, el Estado y el ambiente (Hopwood, 1983; Gray, 1992). A partir de esta serie de desarrollos alternativos, ha surgido la corriente heterodoxa de la contabilidad. Por un lado, esta se muestra despreocupada de las pretensiones cientificistas de las disciplinas. Por otro lado, se muestra muy interesada por el papel de la contabilidad en la vida cotidiana de la gente y por su uso en la toma de decisiones que afectan los procesos de desarrollo (Maunders \& Burritt, 1991), la salud pública (Lowe \& Tinker, 1987), el acceso y calidad de la educación (Mathews, 2001), la estabilidad de las instituciones democráticas (Broadbent \& Guthrie, 2008), la calidad de vida en el trabajo (Mirvis
\& Lawler, 1984), o la sostenibilidad del ambiente (Gray, 1992). Por el carácter público de la multiplicidad de intereses que convergen en la contabilidad, las metodologías de construcción de la disciplina se han diversificado de tal forma que hoy resulta aventurado hacer una ubicación taxonómica de la contabilidad en el campo de las ciencias sociales.

\subsection{La contabilidad en la taxonomía habermasiana}

El conocimiento teórico contable debe su existencia y desarrollo al progresivo perfeccionamiento de las prácticas empíricas, y los instrumentos y técnicas que han hecho posible los procesos de medición, valoración y control de la riqueza en el marco histórico de los modos de producción ${ }^{2}$. Aunque en los medios académicos hay la idea generalizada de que la contabilidad es una disciplina de conocimiento, en la vida diaria de mucha gente común, aún subsiste la percepción de la contabilidad como una técnica registral de conteo y valoración; es decir, para muchos se trata de una práctica lejana o ajena a las discusiones científicas, y más centrada en la mecánica de cargo y abono de las cuentas. A pesar de esta circunstancia, es posible relacionar algunas percepciones académicas de la contabilidad con la taxonomía de las ciencias propuesta por Habermas a través de la relación conocimiento-interés, de la cual se desprenden interesantes conexiones.

Como podrá inferirse, la diversidad y mutabilidad de los objetos de estudio de las disciplinas y los problemas de investigación que de ellos se derivan determinan las metodologías más apropiadas para su estudio e incluso la inserción de tales disciplinas en las categorías taxonómicas propuestas por los diferentes

2 Esto obedece a su origen en la más primitiva racionalidad del conteo y el control, que la arqueóloga Denise Schmandt-Besserat (1999) ubica en las fichas de arcilla descubiertas por ella cerca de Uruk (Irak) y que logró datar hacia 8000 a.C. Tal hallazgo consistiría en la primera manifestación histórica de racionalidad contable, muy anterior a la aparición de la escritura. En ese sentido, en esas fichas contables, se encontraría el origen de la civilización occidental. 
enfoques epistemológicos. Por lo tanto, la preeminencia del trabajo instrumental y el uso del pensamiento analítico en las prácticas contables, y su posterior perfeccionamiento y abstracción en forma de teorías, principios, axiomas y conceptos (Mattessich, 2002; Belkaoui, 2005) describen -conforme con la categorización propuesta por Habermas- el surgimiento de una típica disciplina empírico-analítica. Sin embargo, esa clasificación no es usual ni explícita en la literatura contable.

Posiciones discursivas más avanzadas (o aventuradas) plantearon luego que la contabilidad posee una capacidad de representación o performatividad (Hopwood, 1983; Lowe, 2004; Gray, Adams \& Owen, 2017) que sobrepasa la racionalidad técnico-instrumental. Siguiendo esa línea de pensamiento, se propone que, con apoyo de las metodologías pertinentes, la contabilidad permite interpretar los hechos de la realidad social en función de su inserción en los procesos históricos para dotarlos de sentido como una manifestación de la cultura que caracteriza los usos y prácticas de un pueblo en contacto interactivo con su entorno. Algunos autores como Laughlin (Broadbent \& Laughlin, 1997), Hopwood (1976, 1983, 2009), Gray (1992, 2010), Deegan $(2002,2017)$ y otros han planteado que la contabilidad no siempre debe actuar para resolver los problemas de las organizaciones; como algunos de los problemas escapan a su competencia, conviene abrir espacios para estudiarlos como parte de la realidad, e intentar comprender su estructura y significado como factores de su irresolubilidad:

La urgencia de abordar los problemas del desarrollo sostenible y la falta de creencia con respecto a la posibilidad de su «resolución» nos obliga a desdibujar las fronteras convencionales entre la creación de conocimiento y su aplicación a la política real y la toma de decisiones (Bebbington \& Larrinaga, 2014, p. 410).
El descentramiento del quantum, el abandono de las preocupaciones estadísticas y el nuevo interés por construir narrativas que se derivan de las cifras permitieron que la investigación contable se aventurara en las entrañas de las organizaciones y del mismo sistema capitalista (Hopwood, 1976; Chatfield, 1977; Richardson, 1987) para identificar los intereses económicos, y las intenciones individuales que mueven el sistema empresarial y el aparato estatal, y que determinan los cambios que afectan la vida de los ciudadanos y los colectivos sociales. En esta dinámica, se hizo visible la necesidad de acompañar las metodologías tradicionales de investigación contable con los aportes de algunas ciencias sociales humanas como la sociología, la psicología y la antropología. Estas disciplinas han contribuido para construir el sentido de otra visión de la contabilidad: una que se encuentra adherida a los procesos de modernización de los instrumentos y medios de producción, a los cambios en las formas de trabajo y los modelos de gestión, y a la integración de los conglomerados empresariales y sus efectos en el conjunto de la sociedad: «El desarrollo de un enfoque basado en el discurso de Habermas se puede utilizar para explorar y comprender las organizaciones en un sentido general y no simplemente los sistemas de contabilidad dentro de ellas» (Broadbent \& Laughlin, 1997 p. 640). La CSA es una disciplina que puede reconocerse como ciencia histórico-hermenéutica cuando trasciende el entendimiento para instalarse en el escenario de la comprensión, se adentra en la historia económica y social del entorno en que se desarrolla, interpreta los cambios del entorno desde las estructuras de capital de las empresas y las decisiones que en su interior se toman, puede ver los flujos de dinero como producto de decisiones que agencian intereses de diversa índole, o logra desentrañar el significado de aplicar o no aplicar determinada práctica de una u otra manera.

Contabilidad y Negocios (15) 30, 2020 / ISSN 1992-1896 
Con respecto a estas demarcaciones, debe considerarse que el efecto psicológico de la exposición a la crítica y a la reprobación en una disciplina cuyo ejercicio profesional ha estado adherido a la confianza pública y al reconocimiento, puede hacer que muchos investigadores prefieran no romper con la tradición y conserven posiciones más cautelosas para asegurar su lugar en el establecimiento. Como se verá en detalle más adelante, una posición mucho más arriesgada postula que la contabilidad no solo debe describir, explicar e interpretar; además, debe cuestionar la realidad, y poner en conflicto las ideas, los conceptos, las teorías y los agentes (individuales, corporativos e institucionales) que los representan, para identificar sus divergencias y contradicciones, develar los intereses subyacentes que agencian, y crear el ambiente propicio para las transformaciones que resulten necesarias. Desde esta perspectiva, se asume que vale la pena apostar por provocar los cambios que requiere el mundo contemporáneo. En consecuencia, se presenta una contabilidad enfrentada a la crisis estructural del sistema capitalista, y comprometida con la responsabilidad social (más allá de la filantropía y los postulados de la accountability) y con la justicia ambiental.

Esta caracterización supone la presencia de temas de interés que coinciden con las áreas de trabajo de la CSA, aunque no necesariamente con las formas de trabajo en cada una de ellas. La perspectiva «radical» de esta categorización, aplicada a la CSA, permite considerar este campo de conocimiento como una disciplina crítico-social (Dillard, 1991). Así mismo, es posible visualizar la situación que se presenta en la aplicación de dicho conocimiento al trabajo académico, es decir, se abre un espacio de diálogo y debate para los autores que comparten esta perspectiva epistemológica. Intencionadamente o no, los autores que comparten posiciones teóricas en el tratamiento de una temática terminan conformando una comunidad aun cuando no se conozcan o trabajen juntos, pues la idea de "comunidad» en el ámbito académico y científico se refiere a los intereses y perspectivas (ontológicas, epistemológicas y metodológicas) comunes entre ellos. Esto anima a conocer los vínculos entre las tendencias de pensamiento y los autores por medio de sus productos académicos.

\section{Hallazgos}

\subsection{Tejiendo conexiones entre las ideas y el trabajo}

Tomando en cuenta las anteriores consideraciones taxonómicas de la contabilidad como saber (perspectiva epistemológica), debe reconocerse la relación entre ellas y las formas de trabajo de los investigadores (perspectiva metodológica), lo que permite visualizar claramente algunas sinergias entre tales perspectivas. Una primera percepción de la CSA como saber empírico analítico sustentado en la preeminencia del trabajo humano (Habermas, 1995; Vasco, 1990) encuentra un apropiado canal de realización factual en el enfoque funcionalista, una forma de hacer investigación apegada a la tradición de las técnicas de la contabilidad financiera y orientada a los objetivos de las organizaciones.

De la misma manera, la CSA concebida como disciplina histórico-hermenéutica, y afincada en el uso prioritario del lenguaje como mecanismo de comunicación acerca de la interacción de las organizaciones con la sociedad y el ambiente (Habermas, 1995; Vasco, 1990) encontrará en el enfoque interpretativo la mejor manera de precisar su lectura de la realidad y su construcción de sentido sobre los hechos de los que se ocupa. Desde este enfoque, la investigación en CSA asumirá las acciones y los efectos derivados de la interacción empresa-sociedad-ambiente como un 
fenómeno propio de la realidad social que encarna una creciente complejidad, que puede ser abordada a través de diversos mecanismos dialógicos y hermenéuticos de construcción de sentido y significado.

Quizás, en contraste, la concepción de la CSA como una disciplina crítico-social adherida a las complejas dinámicas y contradicciones de la interacción social (Habermas, 1995; Vasco, 1990) se conecta claramente con el enfoque crítico de la investigación contable, pues en este resulta pertinente cuestionar y confrontar el establecimiento contable, develar la inequidad de la relación empresa-ambiente-sociedad, la debilidad del Estado frente a los organismos reguladores internacionales y a los conglomerados empresariales, y la ineficacia de los acuerdos internacionales en materia de sostenibilidad ambiental. Así, cada concepción de la CSA encontrará una perspectiva epistemológica y metodológica apropiada para desarrollar la investigación en atención a sus premisas conceptuales (ver figura 1). En esa dirección, se configuran y reconfiguran las comunidades académicas y grupos de investigadores.

Figura 1. Enfoques de investigación en CSA

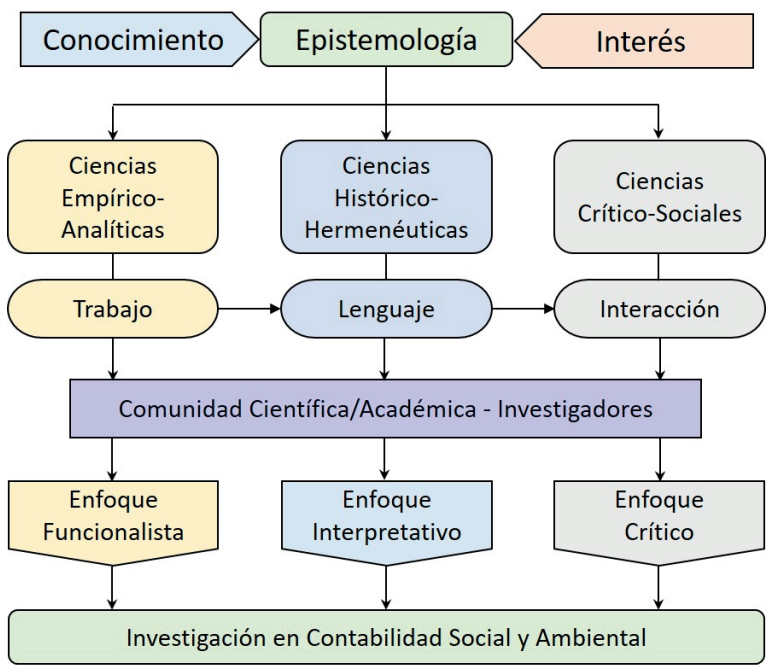

\subsection{Hacia una identidad epistemológica}

La similitud de percepciones acerca de los temas de interés, el uso de marcos conceptuales comunes, la cercanía de los vínculos académicos institucionales y personales, y la correspondencia de las posiciones y argumentos de unos y otros son aspectos que caracterizan a muchos grupos de investigadores en todo el mundo. Así, se facilita su trabajo y se acelera los desarrollos de la ciencia. A pesar de que el concepto kuhniano de comunidad científica involucra elementos adicionales de mayor complejidad, es posible hacer un ejercicio de conexión epistémica entre algunos autores del campo disciplinal de la CSA a partir del análisis de algunos de sus textos más significativos en esta área de conocimiento. Teniendo en cuenta que el análisis de contenido es el mecanismo central del trabajo, se han establecido algunas características (ver tabla 1) que identifican los enfoques de investigación mencionados y que son rastreadas en los textos:

Tabla 1. Categorías de análisis y características asociadas

\section{CATEGORÍAS DE ANÁLISIS Y FACTORES DE IDENTIDAD - Subcategorías}

\begin{tabular}{|l|l|l|}
\hline \multicolumn{1}{|c|}{$\begin{array}{c}\text { C1. Enfoque } \\
\text { funcionalista }\end{array}$} & \multicolumn{1}{|c|}{$\begin{array}{c}\text { C2. Enfoque } \\
\text { interpretativo }\end{array}$} & C3. Enfoque crítico \\
\hline $\begin{array}{l}\text { Apolítico/Aséptico/ } \\
\text { Neutral }\end{array}$ & No resolutivo & $\begin{array}{l}\text { Estudio de con- } \\
\text { flictos }\end{array}$ \\
\hline $\begin{array}{l}\text { Evaluación de } \\
\text { eficiencia }\end{array}$ & $\begin{array}{l}\text { Naturalización } \\
\text { problemática }\end{array}$ & Impulso al cambio \\
\hline Ánimo resolutivo & $\begin{array}{l}\text { Intención com- } \\
\text { prensiva }\end{array}$ & $\begin{array}{l}\text { Foco en relaciones } \\
\text { de poder }\end{array}$ \\
\hline Ahistórico & Histórico & Origen marxista \\
\hline $\begin{array}{l}\text { Positivista/ontoló- } \\
\text { gico/realista }\end{array}$ & $\begin{array}{l}\text { Hermenéutico/ } \\
\text { Sentido }\end{array}$ & $\begin{array}{l}\text { Desigualdad estruc- } \\
\text { tural }\end{array}$ \\
\hline Reduccionista & $\begin{array}{l}\text { Acción contextual } \\
\text { significante }\end{array}$ & $\begin{array}{l}\text { Alienación contra } \\
\text { desarrollo }\end{array}$ \\
\hline
\end{tabular}

Contabilidad y Negocios (15) 30, 2020 / ISSN 1992-1896 


\begin{tabular}{|c|c|c|}
\hline \multicolumn{3}{|c|}{$\begin{array}{l}\text { CATEGORÍAS DE ANÁLISIS Y FACTORES DE } \\
\text { IDENTIDAD - Subcategorías }\end{array}$} \\
\hline $\begin{array}{l}\text { C1. Enfoque } \\
\text { funcionalista }\end{array}$ & $\begin{array}{l}\text { C2. Enfoque } \\
\text { interpretativo }\end{array}$ & C3. Enfoque crítico \\
\hline $\begin{array}{l}\text { Metodologías cuan- } \\
\text { titativas }\end{array}$ & $\begin{array}{l}\text { Construcción } \\
\text { social significante }\end{array}$ & $\begin{array}{l}\text { Falsa neutralidad } \\
\text { del mercado }\end{array}$ \\
\hline $\begin{array}{l}\text { Optimista sobre el } \\
\text { progreso }\end{array}$ & $\begin{array}{l}\text { Transformación de } \\
\text { significados }\end{array}$ & $\begin{array}{l}\text { Consumo influido/ } \\
\text { manipulable }\end{array}$ \\
\hline $\begin{array}{l}\text { Escéptico sobre lo } \\
\text { humano }\end{array}$ & $\begin{array}{l}\text { Práctica contable } \\
\text { intencionada }\end{array}$ & $\begin{array}{l}\text { Valor social del } \\
\text { trabajo }\end{array}$ \\
\hline $\begin{array}{l}\text { Claridad/rigurosi- } \\
\text { dad conceptual }\end{array}$ & $\begin{array}{l}\text { Información/Len- } \\
\text { guaje: contextual }\end{array}$ & $\begin{array}{l}\text { Totalidad da sen- } \\
\text { tido a partes }\end{array}$ \\
\hline $\begin{array}{l}\text { Escéptico/No espe- } \\
\text { culativo }\end{array}$ & $\begin{array}{l}\text { Significados objeti- } \\
\text { vizantes }\end{array}$ & $\begin{array}{l}\text { Estado no corrige al } \\
\text { mercado }\end{array}$ \\
\hline $\begin{array}{l}\text { Pretensión demos- } \\
\text { trativa }\end{array}$ & $\begin{array}{l}\text { Falibilidad de las } \\
\text { prácticas }\end{array}$ & $\begin{array}{l}\text { Crisis consubstan- } \\
\text { cial sistémica }\end{array}$ \\
\hline $\begin{array}{l}\text { Instrumentos mate- } \\
\text { máticos }\end{array}$ & $\begin{array}{l}\text { Prácticas racionali- } \\
\text { zantes }\end{array}$ & $\begin{array}{l}\text { Contabilidad racio- } \\
\text { naliza el cálculo }\end{array}$ \\
\hline $\begin{array}{l}\text { Obsesión por la } \\
\text { exactitud }\end{array}$ & $\begin{array}{l}\text { Técnicas socioló- } \\
\text { gicas }\end{array}$ & Unicidad contable \\
\hline Precisión/Certeza & $\begin{array}{l}\text { Manipulación de } \\
\text { instrumentos }\end{array}$ & $\begin{array}{l}\text { Contabilidad legi- } \\
\text { tima la desigualdad }\end{array}$ \\
\hline $\begin{array}{l}\text { Presunción de } \\
\text { objetividad }\end{array}$ & $\begin{array}{l}\text { Interpretación para } \\
\text { interpretar }\end{array}$ & $\begin{array}{l}\text { Espíritu denun- } \\
\text { ciante }\end{array}$ \\
\hline
\end{tabular}

Fuente: Elaboración propia sobre la base de Burrell y Morgan (1979), y Larrinaga (1999).

Al hacer el análisis de los textos, queda claro que la investigación de los autores más críticos es tan importante y útil como la que hacen los investigadores más tradicionales. La diferencia está en el tipo de problemas que abordan, los objetivos que se plantean y las metodologías que utilizan para alcanzarlos. Aunque la promoción de la sostenibilidad y la importancia de reportar las acciones de las organizaciones en este aspecto es una constante en todos los autores de CSA, esta sostenibilidad se asume de diferentes maneras dependiendo del enfoque utilizado. El rastreo de estas características o rasgos de identidad epistemológica arroja como resultado las siguientes conexiones que se han agrupado en las siguientes secciones.

\subsubsection{Enfoque funcionalista: el rastro de la tradición}

Debido a las diferencias de percepción encontradas en los textos, el reporte de «sostenibilidad» como concepto y como instrumento tiene entre los autores más tradicionalistas una connotación funcional prioritaria que se expresa en la necesidad de aplicar un conocimiento contable a las acciones de sostenibilidad para informar acerca de ello, esto sucede en cualquier otro campo de acción organizacional en el que se produce información útil para la toma de decisiones empresariales:

Los «costes sociales» pueden expresarse como «cargos efluentes» impuestos contra la fuente de la contaminación. Estos últimos, que en efecto resultan previamente de la «internalización» de los impactos de costos «externos», luego ingresan en los cálculos gerenciales de una empresa individual como cualquier otro coste de hacer negocios (Dierkes \& Preston, 1977, p. 20).

Así, surge la CSA empresarial como un objeto de gestión, evaluación y control. Un primer grupo de observaciones deja en claro la necesidad de evaluar la eficiencia de la inversión aplicada a la resolución de problemas concernientes a las comunidades relacionadas con las empresas y el medio físico. Aun así, no es visible ningún interés por la intencionalidad que orienta tales acciones. Por ejemplo, Qiu, Shaukat y Tharyan (2016) aseguran lo siguiente:

En todas las industrias, el desempeño social y su divulgación son importantes para los inversionistas, pues pueden ayudar a las empresas a obtener beneficios económicos reales. Nuestro hallazgo de la tasa de crecimiento esperada de los flujos de efectivo como impulsores del precio de las acciones de las empresas de alta divulgación social apoya aún más esta última afirmación (p. 113).

En los textos de esta tendencia, es muy importante la medición cuantitativa y la valoración económica de aspectos como los impactos sociales y ambientales 
provocados en la producción, los costos de la gestión ambiental de esos impactos, las erogaciones por acciones y prácticas de responsabilidad social corporativa, el efecto que tales valoraciones tiene en la estructura financiera de las organizaciones, y la revelación que de esas mediciones y valoraciones hagan en sus informes de sostenibilidad. Quizás por eso, las principales preocupaciones son vincular la información social de las empresas con su desempeño económico (Dierkes \& Preston, 1977) e identificar niveles de involucramiento financiero en dichas acciones (Preston, O'Bannon \& Douglas, 1997). Así, se presta especial atención a los costes en que se incurre para su ejecución (Epstein, 1996), evidenciados en la precisión matemática que muestran los cálculos financieros.

En los primeros textos analizados, las variables financieras envolvían las propuestas para medir con precisión y valorar eficientemente las acciones empresariales orientadas a la comunidad relacionada y al medio natural (Belkaoui \& Karpik, 1989), aunque es claro que este último elemento no era una prioridad tan valorada como ahora. A esas apreciaciones se ańadían otras variables económicas para determinar el curso de las decisiones gerenciales en torno a la divulgación de información social mediante modelos estadísticos (Epstein \& Freedman, 1994). Esto pudo ser probado por los mismos autores en muchas compañías industriales de Estados Unidos y el Reino Unido, aunque tomaron la precaución de no generalizar sus resultados. Por otro lado, en estos estudios, la perspectiva política de la acción social de las organizaciones -incluso en las concernientes al sector público- es prácticamente inexistente o, al menos, no es fácilmente perceptible. Entonces, se deja a la contabilidad en una situación indefinida en la relación empresa-sociedad. En esta circunstancia, resulta más claro verla como un sistema de información al servicio de la organización antes que como una ciencia social en ciernes.

El carácter voluntario de la divulgación social, ética y ambiental, y los problemas del relativismo ético, han dejado a las empresas en una situación de incertidumbre [...] están recurriendo a sus inversionistas principales para obtener asesoramiento sobre divulgación pública social, ética y ambiental, a través de la divulgación privada social, ética y ambiental (Solomon $\&$ Solomon, 2006, p. 585).

En contraste, la exposición de los temas y la descripción de los problemas son muy claras y precisas y se soportan en datos e indicadores tomados directamente de las fuentes, lo que favorece la comprensión de los lectores. Las metodologías utilizadas son variadas, aunque siempre de orden cuantitativo con fuertes componentes estadísticos (Preston, 1978; Belkaoui \& Karpik, 1989; Qiu, et al., 2016); estos aspectos ofrecerían fiabilidad por la rigurosidad de los cálculos y la exactitud de los resultados.

En líneas generales, los hallazgos y resultados están referidos a interrelaciones entre variables económicas y socioambientales, definidas por el interés en los beneficios (Epstein \& Friedmann, 1994; Peloza, 2009, Qiu, 2016), y propuestas de mejoramiento de la gestión por la vía de la optimización de los controles, la reducción de los impactos — particularmente en la industria(Preston, 1978) y la consecuente reducción de costes. En líneas generales, los rasgos característicos del enfoque funcionalista son fácilmente visibles en los textos de Ahmed Belkaoui, Marc J. Epstein, Lee Preston, John Peloza, Aris Solomon, Yan Qiu. y muy tangencialmente, en los de Reg Mattews, cuyos trabajos incursionan en el enfoque interpretativo.

Las consideraciones finales de estos artículos coinciden en otorgar mucha confianza a la lógica empresarial y a las fuerzas del mercado:

Contabilidad y Negocios (15) 30, 2020 / ISSN 1992-1896 
En muchos de estos estudios, parece que la divulgación de información social provocó una reacción del mercado. Los investigadores dedujeron de esto que los inversionistas estaban usando información social para tomar decisiones de inversión y, por lo tanto, debería haber demanda de revelaciones sociales (Epstein \& Freedman, 1994, p. 107).

Al mismo tiempo, muchos de ellos presentan las actitudes y decisiones de los administradores, propietarios, inversionistas, clientes y proveedores (Solomon $\&$ Solomon, 2006), como un factor de riesgo, que es preciso conocer, estudiar, medir y controlar.

Es innegable que los trabajos que hemos agrupado en este enfoque funcionalista dan cuenta de una alta preocupación por el uso adecuado de las herramientas estadísticas, el acertado diseño de los modelos matemáticos que sustentan las mediciones, la racionalidad de los resultados, el apego a la normativa técnica internacional, la comparabilidad de la información emitida, y por supuesto, la orientación de las acciones de gestión social y ambiental a los objetivos empresariales de rentabilidad. Visto así, estas consideraciones auguran larga vida a la investigación contable en la mainstream, y aseguran el apoyo político y económico de las grandes corporaciones y gremios profesionales que la hacen posible en todo el mundo.

\subsubsection{Enfoque interpretativo: la reinvención de la realidad}

La mayor cantidad de textos examinados presentan características referidas a cuestiones interpretativas, a la construcción de significados a través del lenguaje de la información contable y a la intención comprensiva de la realidad organizacional. En ellos se observan las organizaciones como artefactos y dispositivos propios del modo de producción capitalista. Asimismo, se muestra un esfuerzo por describir, categorizar, analizar e interpretar las prácticas contables como ejercicios de naturalización de las inequidades producidas por las fuerzas del mercado, las regulaciones, las formas de trabajo y las decisiones gerenciales. Los textos agrupados en esta categoría definen un perfil interpretativo de los autores, aunque con matices próximos a otros enfoques: «Los enfoques interpretativos generalmente enfatizan la forma en que la historia y la indexación de una situación actúan de manera reflexiva para producir una «realidad particular» (Broadbent $\&$ Laughlin, 1997 p. 643). Tal realidad, derivada del lenguaje, termina por cambiar la contabilidad.

Aunque resulte obvio y la anotación parezca tardía, debe decirse que la idea misma de CSA implica también una mutación del sentido tradicional del concepto de contabilidad (Gray et al., 2017). Esta transformación se debe a la introducción de una perspectiva -mediada por el lenguaje- que reinterpreta el papel de la disciplina en el contexto del desarrollo y constituye, una nueva visión de este saber en relación con la sociedad y el ambiente ${ }^{3}$. Dicha idea tomaría nueva forma en tiempos de sostenibilidad y globalización.

Al ser el eje transversal de este campo disciplinar, la sostenibilidad ha marcado el desarrollo no solo de un amplio cuerpo de literatura, sino también de una nueva forma de expresar, interpretar y representar las relaciones entre las organizaciones, el Estado, la socie-

3 La idea misma de "ambiente» ha sufrido algunas transformaciones en función de la interpretación y del uso que algunos autores han hecho de la hermenéutica como metodología del trabajo académico. Los cambios también se han producido a partir de las traducciones desde las lenguas originales (Evans, 2018) de los textos clásicos. En inglés, las ideas de environment y nature se asocian a todos los aspectos del entorno, mientras en español aún subsisten discusiones sobre «ambiente» $\mathrm{y}$ «medio ambiente», o «naturaleza» $\mathrm{y}$ «medio natural». 
dad y el ambiente. Quizás los profesores Mathews y Gray son los pioneros de esta tendencia de pensamiento que cambió para siempre la disciplina: "en algún momento de la década de 1990, comenzamos a ver a las organizaciones hablando sobre 'cuentas de sostenibilidad', este giro lingüístico, deliberadamente o no, atrajo nuestra atención a una escala global" (Gray, 2010, p. 48). Desde entonces, el trabajo de Gray desde CSEAR - University of St. Andrews marcó el derrotero de la CSA y ha expandido la perspectiva heterodoxa de la disciplina por todo el mundo.

El trabajo intelectual en el campo de la CSA implica pensar y, sobre todo, repensar el papel de las organizaciones, y del modo de producir y reproducir riqueza en este tiempo. Tales replanteamientos ponen en tela de juicio la racionalidad del orden económico mundial (Piketty, 2014) y de la propia forma de vida de los humanos. En este sentido, Gray (2010) advertía que «si abrazamos tal nivel de duda en nuestros apegos ontológicos y epistemológicos, es posible que debamos aceptar de manera más explícita que las cuentas de sostenibilidad comunicarán y construirán la realidad» (p. 48). Se trata de un argumento que plantea una nueva visión de la contabilidad, reconstruida por el lenguaje más allá de los hechos y las cifras. Esta visión ha sido retomada por un crecido número de investigadores y por eso el trabajo de algunos de ellos es objeto de análisis en este trabajo.

Más allá de la distancia que separa la tradición de la contabilidad financiera y los desarrollos de la CSA (Hopwood, 2009), este último campo de conocimiento también ha sido finalmente colonizado por la racionalidad financiera de las corporaciones: «la captura por grupos dominantes es evidente en algunos elementos del proyecto de Contabilidad Ambiental. [...] Esta parte de la Contabilidad ambiental no aborda preocupaciones más amplias ni la estructura en la que existen las prácticas comerciales y contables» (Bebbington, 1997, p. 388). Cada vez resulta más difícil para los investigadores distinguir las intencionalidades que subyacen en los reportes de sostenibilidad de las empresas, pues las palabras y expresiones utilizadas configuran significados intencionados (Cho \& Patten, 2007) que transforman la percepción que los ciudadanos tienen de las corporaciones y sus acciones (Moneva, Archel \& Correa, 2006), el propósito de sus revelaciones y la validación de sus efectos a la luz de los preceptos de gestión sostenible, contenidos en los acuerdos nacionales y multilaterales. Sin embargo, hoy casi nadie duda de que la contabilidad es un factor fundamental para el sostenimiento del orden económico mundial (Bebbington, 1997, p. 375) y de las inequidades que de él se derivan (Spence, Husillos $\&$ Correa, 2010).

Este fenómeno no solo es visible en la información que producen las empresas, sino que se ha instalado en las universidades, de forma que la educación contable también se ha contaminado de esta perspectiva utilitarista del conocimiento. «La forma en que se concibe, se construye y se enseña la contabilidad hace inevitable que los estudiantes de contabilidad experimenten atrofia intelectual y moral» (Mc Phail, Bebbington \& Gray, 1994, p. 3), lo cual preocupa mucho a los académicos e investigadores. En este sentido, debe considerarse además, el papel de los organismos reguladores privados que actualmente promueven la imposición de un modelo estandarizado de educación contable en todo el mundo, sobre la base de eliminar la formación sociohumanística —imprescindible para la investigación en ciencias sociales - en favor de la técnica contable y la racionalidad financiera (Gómez \& Sarmiento, 2016).

La construcción de significados acerca de la información contable es una tarea que se sobrepone al 
significado que esta información ha construido de las prácticas contables. A su vez, estas prácticas son una forma de representar los hechos de la actividad corporativa. Por ello, la realidad adquiere un carácter relativizado por el lenguaje y el interés de quienes construyen la información y los significados (Broadbent $\&$ Laughlin, 1997). En atención a esto y a las emergencias de la época, muchos de los autores han centrado su trabajo de investigación interpretativa en contextos y situaciones específicas (Unerman \& O’Dwyer, 2006; Ball \& Bebbington, 2008). Por eso, abundan los estudios de caso acerca del desempeño socioambiental corporativo en sectores económicos muy localizados (Larrinaga, Carrasco, Correa, Llena \& Moneva, 2002) o afectados por fenómenos nacionales ( $\mathrm{O}^{\prime} \mathrm{D}$ wyer, Owen \& Unerman, 2011); y organizaciones (ONG) cuyo desempeño escapa a la lógica empresarial, pero ameritan una mirada desde las premisas conceptuales de la hermenéutica (Unerman \& O’Dwyer, 2006) y las técnicas del análisis sociológico.

Dentro de los hallazgos que ha ofrecido la investigación en el enfoque interpretativo, se destaca la revelación de intencionalidades subyacentes en los reportes de sostenibilidad publicados por las empresas. Tales ejercicios revelan el afán de los directivos y las corporaciones por legitimarse ante la opinión pública (O’Dwyer et al., 2011; Deegan, 2002; Cho \& Patten, 2007; Archel et al., 2011), especialmente en algunos contextos específicos donde suele ser muy aguda e influyente en sus percepciones sobre la actuación de las empresas:

Tal motivación para informar (para legitimar las operaciones de organización) estaría en contraste con un enfoque de presentación de informes que refleje la aceptación por parte de los gerentes, de un compromiso o responsabilidad de divulgar información a aquellos que tienen derecho a conocerla (Deegan, 2002, p. 283).
En ese sentido, es innegable que las empresas buscan protegerse de reacciones adversas en los mercados de valores. El desarrollo de la investigación en este enfoque, ha abierto camino a nuevas inquietudes que se traducen en abordajes no previstos en la perspectiva tradicional de la contabilidad (Lehman \& Ockabol, 2005; Archel et al., 2011; Brown \& Dillard, 2013) y que cuestionan los pilares mismos del sistema capitalista. Esta dinámica ha llevado a que algunos autores más tradicionales como Chua (1986), Baker (2005), y Hopper, Lassou y Soobaroyen (2017) cuestionen la «ideologización de la disciplina»: en su criterio, debería ser neutralizada para no restarle carácter científico. Sin embargo, autores más progresistas vienen impulsando desde tiempo atrás una perspectiva de investigación aún más crítica. Dicha tendencia se encuentra orientada a desentrañar los secretos que oculta la contabilidad. Asimismo, busca develar los intereses políticos de los organismos reguladores, la doble moral de muchas empresas que perpetúan el modelo de producción y consumo exponencial, la racionalidad predadora de los ecosistemas, y las relaciones de inequidad e injusticia social que imperan en el planeta.

\subsubsection{Enfoque crítico: conflicto y transformación}

Tomando en cuenta el importante rol que cumple la CSA en la captura y procesamiento de información referida al comportamiento de las empresas, se hizo evidente que esta función es un avance para la racionalización, modernización y democratización de los mecanismos de acceso a la información. Sin embargo, tales representaciones y su interpretación por sí mismas no generan cambios significativos en las relaciones entre las empresas y la sociedad. Lehman (2006) afirma que en sus «intentos por crear un cambio social a través de supuestos racionales, [la contabilidad] perpetúa una estrategia que permanece atrapada dentro 
de los confines del presente» (p. 759). Esto sugiere la necesidad de poner en conflicto la institución contable (Puxty et al., 1987) como el pilar que ha sido en el sostén del capitalismo occidental.

De esta perspectiva epistemológica -si se quiere, más radical- del pensamiento contable contemporáneo, ha surgido un enfoque de investigación crítico, apoyado en metodologías cualitativas de origen sociológico. En este enfoque, debe destacarse el trabajo del profesor Tony Lowe como pionero de la Escuela de Scheffield, pues sus aportes abrieron camino a propuestas más audaces que han generado significativos cambios en la forma de investigar, interpretar y evaluar la información relativa al comportamiento empresarial (Dillard, 2014). El enfoque crítico de la investigación en CSA se asocia al estudio de las problemáticas estructurales de la economía mundial (Piketty, 2014) y sus efectos sobre las sociedades (Cooper \& Puxty, 1996). Además, se vincula con los problemas cotidianos de amplios sectores de la población afectados por las políticas de los gobiernos y la acción de las empresas (Lehman \& Ockabol, 2005), y de ecosistemas vulnerables (Laine, 2005; Ball \& Bebbington, 2008) que están en riesgo por cuenta de esas prácticas y agentes.

La literatura examinada muestra que, recientemente, se ha incrementado el trabajo contable en temas sociales clave, como la reducción de las desigualdades, la reivindicación de las libertades civiles, la responsabilidad penal de las organizaciones (compliance), la lucha contra la corrupción, la defensa de los derechos humanos, la erradicación de la esclavitud moderna y la trata de personas, la defensa de las minorías étnicas y pueblos indígenas, y la opacidad de la información de las corporaciones frente a sus grupos de interés. De la misma forma, ha crecido la promoción de mecanismos de participación social para presionar los cambios legislativos, y las transformaciones estructurales que la actual situación social y ambiental demanda. Los reclamos en el campo ambiental incluyen como prioridades de estudio los conflictos ambientales generados por sectores como minería, hidrocarburos, energía nuclear, pesca y caza marina, explotación forestal, gestión de residuos y desechos, y educación ambiental.

Más allá de los aportes, los textos analizados argumentan que la investigación en CSA es aún incipiente y no ha explorado todo su potencial (Adams \& Larrinaga, 2007), pero puede ser un instrumento que les permita a los ciudadanos construir autonomía para reducir la inequidad que domina el escenario de la relación empresas-comunidad-ambiente. A pesar de las fuertes críticas a la investigación en CSA (Tinker et al., 1991; Brown \& Dillard, 2013) por su alineación con los intereses corporativos, por su función legitimadora de las desigualdades sociales y por la debilidad de su propio discurso frente al poder económico, aparecen voces como la de Spence (2009), que ofrece opciones de cambio para la investigación en la CSA: «la contabilidad social podría ser una práctica clave para sacar a la sociedad civil de su letargo consumista, inducido por las relaciones públicas, y para formar un poder compensatorio» (p. 223).

La complejidad de los problemas sociales y su estrecho vínculo con las relaciones de poder económico han abierto un enorme abanico de posibilidades de investigación en CSA. La transición del capitalismo industrial al capitalismo financiero y la crisis que este tránsito supone han profundizado la brecha entre las sociedades postindustriales, fundamentadas en la oferta de servicios especializados y la gestión tecnológica de la información y las que aún luchan por consolidar sus aparatos productivos sobre la base del uso intensivo de recursos naturales y el trabajo de la población (Piketty, 2014). Actualmente, la CSA se enfrenta a estudiar la forma en que los gobiernos, las 
corporaciones y las ONG pueden contribuir a mitigar los efectos de la crisis estructural del capitalismo sobre las poblaciones y los ecosistemas (Lehman, 2001). Cada vez es más evidente que los ritmos de acumulación de la riqueza producen brechas de crecimiento y desarrollo, que se traducen en desempleo, precarización (y esclavitud) en el trabajo, flujos migratorios (Lehman, Annisette \& Angyemang, 2016), rezago educativo y tecnológico, violencia de género, corrupción del Estado, diversas formas de discriminación social, y múltiples vectores de degradación ambiental.

Un grupo de textos coincide en señalar que la CSA es políticamente débil (Tinker et al., 1991). Además, señalan que está lejos de cumplir con una misión trasformadora de la sociedad actual, pues muchas de las metodologías de medición y reconocimiento reproducen la lógica del establecimiento; por su parte, los informes de sostenibilidad se han estandarizado en torno a la retórica del discurso empresarial (Cho, Laine, Roberts \& Rodrigue, 2015; Archel et al., 2011) para encubrir sistemáticamente prácticas alejadas de la ética, y acciones discriminatorias e incluso predatorias. Por ello, hay llamados para ampliar el radio de acción de estas discusiones: «Los debates sobre el compromiso de la CSA deben tener lugar no solo entre académicos y 'líderes' políticos, de negocios o de ONG, sino también en comunidades más amplias que a las que todos pertenecemos» (Brown \& Dillard, 2013, p. 16). Esto se fundamenta en que los problemas que de allí emergen atañen a todo el conjunto de la sociedad. Así, la contabilidad como conocimiento y práctica de legitimación de la desigualdad es el rasgo más común en la perspectiva crítica de los documentos analizados, por lo que algunos autores reclaman la aplicación de teorías y metodologías más contundentes para resignificar la información publicada en los reportes y revelar los intereses ocultos tras ellos, y de esta manera, rescatar la CSA de la ineficacia social y la inocuidad política (Spence, Husillos \& Correa, 2010).

En la perspectiva crítica de la investigación en CSA, hay autores que insinúan la necesidad de que sus investigadores abandonen la comodidad del discurso hegemónico y homogeneizante (Tinker \& Gray, 2003; Spence, 2009; Archel et al., 2011) que finalmente reproduce las lógicas que dice combatir. Esto está provocando diferencias dentro de la misma heterodoxia, pues se cree que esta corriente ha sido cooptada por la lógica normativa de las organizaciones internacionales que promueven el desarrollo sostenible (Bebbington \& Unerman, 2018); de esa forma, el propio trabajo académico, aunque valioso y riguroso, no contribuye efectivamente a generar los compromisos y las transformaciones que la sociedad demanda de manera urgente. Entonces, se infiere con facilidad que los autores más críticos de este enfoque abogan por un trabajo más comprometido con la reducción de las desigualdades sociales, y los impactos ambientales que genera y promueve el modo de producción. A la vez, impulsan agendas de investigación más incluyentes, que permitan la autocrítica; el fortalecimiento teórico; y el relevo generacional de esta comunidad, en la que está sembrada la esperanza del cambio.

\subsection{En busca de la comunidad científica}

Además de la necesaria comunión epistemológica, el rasgo identitario más característico de las comunidades científicas es la cohesión, pues el hecho de que un autor reconozca el trabajo de otros como referente para el suyo y que lo haga de forma recurrente da cuenta de la reciprocidad de sus posiciones argumentativas y de su conexión personal. Hace décadas, este fenómeno era producto de la cercanía física de los investigadores. Por lo tanto, era común que la aparición de escuelas y corrientes de pensamiento se diese 
en torno a algunos maestros en determinadas universidades y centros de investigación, donde se agrupaban las élites académicas de la época. Los autores analizados en este trabajo muestran que, aunque existen algunos núcleos de interés académico, la proximidad física no es condición necesaria ni suficiente para la identidad epistémica. Los textos/autores analizados en este trabajo provienen de universidades del Reino Unido (13), Estados Unidos (6), Australia (6), España (5), Nueva Zelanda (2), Canadá (2) y Finlandia (1). Sin embargo, la comunidad se encuentra en permanente expansión gracias a los procesos de investigación en programas de doctorado, y la socialización de sus productos en conferencias, congresos e importantes publicaciones internacionales.

Por supuesto, esta es una mirada limitada y provisional, pues resulta imposible estudiar toda la obra de cada autor en este espacio, así como incluir a todos los autores que trabajan actualmente en la temática de la CSA. Sin embargo, este ejercicio ha permitido visualizar afinidades conceptuales entre los autores seleccionados (Figura 2). De esta manera, se busca abrir camino a la articulación de una comunidad científica en esta área de conocimiento.

\section{Consideraciones finales}

Ante la imposibilidad de plantear conclusiones en una discusión que sigue abierta, debe reconocerse que este ejercicio es válido y útil, pero no suficiente para hacer una ubicación categórica de los autores en determinado enfoque teórico o una perspectiva metodológica específica. Esta afirmación se fundamenta en al menos tres razones: a) el movimiento oscilatorio que presenta el lenguaje de un mismo autor (base de este análisis) en la producción de uno a otro texto; b) la constante aparición de nuevos textos que podrían o deberían

Figura 2. Enfoques epistemológicos y afinidades conceptuales

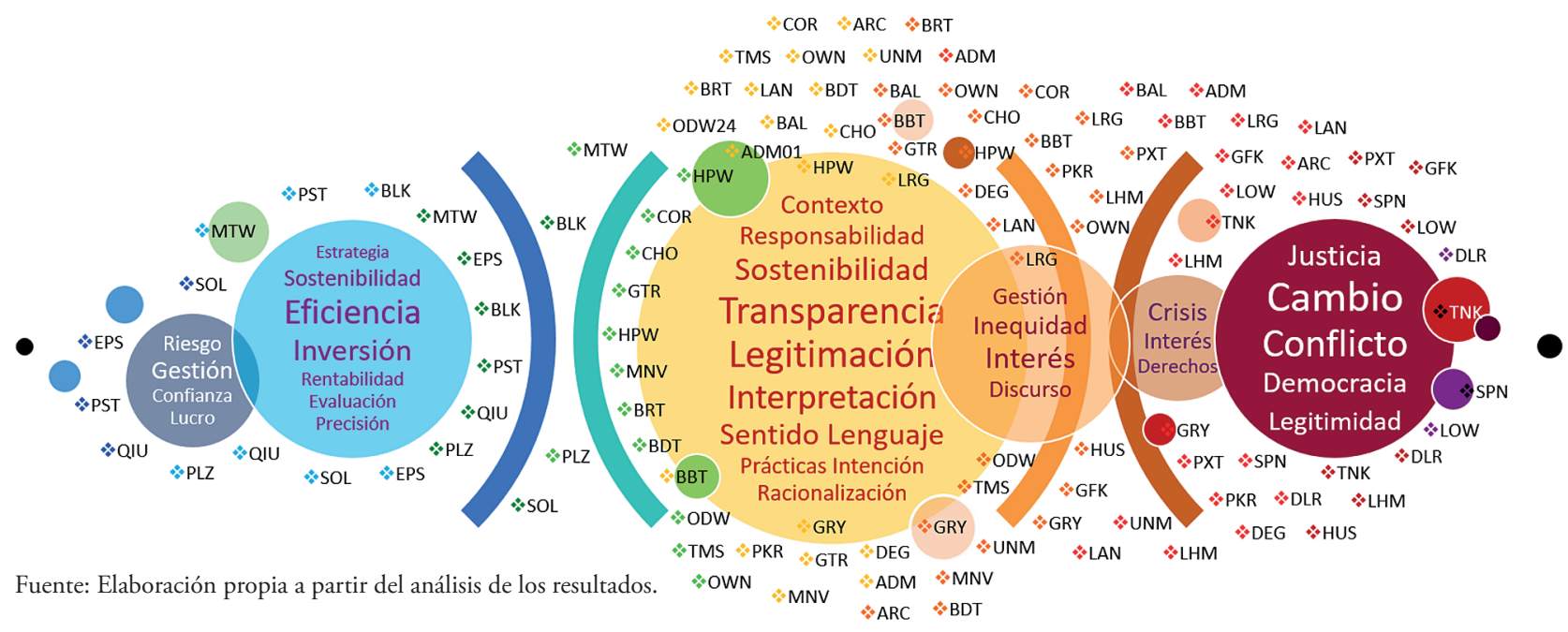

E. Funcionalista

C*. Empírico-Analíticas
E. Interpretativo

C*. Histórico-Hermenéuticas
E. Crítico

C*. Crítico-Sociales 
ser considerados en este análisis; y c) la influencia que tienen otros autores sobre ellos cuando trabajan en coautoría, lo que es cada vez más común dadas las facilidades tecnológicas y la creciente movilidad de los académicos entre países. Más allá de las limitaciones, este trabajo ha logrado evidenciar numerosos rasgos de identidad entre los autores, que permiten afirmar que sí existe una comunidad científica —aún más grande de lo que muestra este ejercicio- en el campo de la CSA, y que su dinámica augura crecimiento y consolidación. El solo hecho de que el rastreo no incluya a otros autores muy reconocidos abre la posibilidad de que haya muchos más puntos en común con otros grupos de investigadores alrededor del mundo.

Si bien los enfoques propuestos para esta caracterización tienen rasgos conceptuales claramente diferenciados, la ubicación de los autores en sus categorías no resultó pertinente, pues son los temas de sus productos los que se movilizan entre las categorías de dichos enfoques. El límite entre el enfoque funcionalista y el interpretativo define por sí mismo la frontera entre la ortodoxia y la heterodoxia contable, pero podría hacerse más difuso si se consideran algunas paradojas ${ }^{4}$ presentes incluso en publicaciones ubicadas en la médula de la mainstream. De igual manera, la frontera entre los autores interpretativos, que son mayoría, y los que han sido vinculados al enfoque crítico es muy difusa. Por ello, resulta bastante difícil determinar su ubicación de manera categórica. Esta idea retrata de buena forma el eclecticismo propio de las vertientes alternativas, que por naturaleza son menos conservadoras y más proclives al cambio.

Como una contribución final, este trabajo puede cumplir la función didáctica de facilitar el estudio de la CSA desde sus bases epistemológicas. Los esquemas aprehensivos-comprensivos en esta área de conocimiento social son cada vez más provisionales (e, incluso, limitados) debido a la complejidad propia de los problemas socioambientales. Desde esta perspectiva, se espera propiciar un diálogo más amplio a partir de este trabajo, que está abierto — como debe ser- al debate, la crítica y la rectificación.

\subsection{Limitaciones y prospectiva}

Las principales limitantes de este estudio son las siguientes: 1) este trabajo solo incluye literatura publicada en inglés ${ }^{5}$ y de un número reducido de revistas, aunque se traten de las más reconocidas del mundo en este campo; y 2) el número de autores seleccionados, que podría ampliarse en un próximo estudio; incluso, podrían considerarse las apreciaciones de los autores con respecto a la CSA con el fin de dar mejor cuenta de este campo disciplinar y construir un horizonte de comprensión que permita transitar de los inevitables problemas a las deseadas soluciones.

4 Los tres enfoques que se han utilizado como categorías de análisis (funcionalista, interpretativo y crítico) incorporan dualidades muy complejas del pensamiento occidental. Mauricio Gómez (2006) revela posiciones tan paradójicas como que, por ejemplo, «la economía neoclásica y la economía financiera son profundamente idealistas: sus premisas metodológicas declaran mercados perfectos, asumen perfecta información, inmovilizan fuerzas del mercado, etc., condiciones que de hecho no existen, pero que son usadas de cabo a rabo por los funcionalistas, que se consideran a sí mismos como realistas en sentido ontológico y positivistas en términos epistemológicos».

5 Aunque las publicaciones académicas de la mayoría de las disciplinas tienden a publicarse y difundirse en inglés, la dinámica investigativa de la CSA en otras lenguas, como el español o el portugués, también tiene importantes desarrollos en comunidades académicas que empiezan a llamar la atención de organismos como CSEAR y Critical Perspectives on Accounting, pioneras en conectar sus procesos de producción y discusión con los de Iberoamérica. 


\section{Referencias}

Adams, C. A. (2002). Internal Organizational Factors Influencing Corporate Social and Ethical Reporting: Beyond Current Theorizing. Accounting, Auditing \& Accountability Journal, 15(2), 223-250. https://doi. org/10.1108/09513570210418905

Adams, C. \& Larrinaga, C. (2007). Engaging with Organizations in Pursuit of Improved Sustainability Accounting and Performance. Accounting, Auditing and Accountability Journal, 20(3), 333-355. https:// doi.org/10.1108/09513570710748535

Aktouf, O. (2010). Visión crítica de la teoría de la gestión estratégica. Teuken Bidikay, 1(1), 13-32. https://doi. org/10.33571/teuken

Aktouf, O. (2011). La metodología de las ciencias sociales y el enfoque cualitativo en las organizaciones. Cali: Universidad del Valle.

Archel, P., Husillos, J. \& Spence, C. (2011). The Institutionalization of Unaccountability: Loading the Dice of Corporate Social Responsibility Discourse. Accounting, Organizations and Society, 39, 395-413. https:// doi.org/10.1016/j.aos.2011.06.003

Ariza, D., León, F. \& Gómez, M. (2006). Una aproximación a los perfiles ambientales de la empresa. Innovar, 16 (28), 57-74. https://doi.org/10.15446/innovar

Baker, R. C. (2005). What is the Meaning of the "Public Interest": Examining the Ideology of the American Public Accounting Profession. Accounting, Auditing \& Accountability Journal, 18(5), 690-703. https://doi. org/10.1108/09513570510620510

Ball, A. \& Bebbington, J. (2008). Accounting and Reporting for Sustainable Development in Public Service Organizations. Public Money and Management, 28(6), 323-326. https://doi.org/10.1111/j.1467-9302.2008.00662.x

Ball, A., Broadbent, J. \& Jarvis, T. (2006). Waste Management, the Challenges of the PFI and Sustainability Reporting. Business Strategy and the Environment, 15, 258-274. https://doi.org/10.1002/bse.532
Bebbington, J. (1997). Engagement, Education and Sustainability. A Review Essay on Environmental Accounting. Accounting, Auditing and Accountability Journal, 10(3), 365-381. https:// doi.org/10.1108/09513579710178115

Bebbington, J. \& Gray, R. (2001). An Account of Sustainability: Failure, Success and a Reconceptualization. Critical Perspectives on Accounting, 12, 557-587. https://doi.org/10.1006/cpac.2000.0450

Bebbington, J. \& Larrinaga, C. (2014). Accounting and Sustainable Development: An Exploration. Accounting, Organizations and Society, 39, 395-413. https:// doi.org/10.1016/j.aos.2014.01.003

Bebbington, J., Larrinaga, C. \& Moneva, J.M. (2008). Corporate Social Reporting and Reputation Risk management. Accounting, Auditing \& Accountability Journal, 21(3), 337361, https://doi.org/10.1108/09513570810863932

Bebbington, J. \& Thomson, I. (2007). Social and Environmental Accounting, Auditing, and Reporting: A Potential Source of Organizational Risk Governance? Environment and Planning C. Government and Policy, 25, 38-55. https://doi.org/10.1068/c0616j

Bebbington, J. \& Unerman, J. (2018). Achieving the United Nations Sustainable Development Goals: An Enabling Role for Accounting Research. Accounting, Auditing \& Accountability Journal, 31(1), 2-24. https://doi.org/10.1108/AAAJ-05-2017-2929

Belkaoui, A. (2005). Accounting Theory. 5a ed. Chicago: Thomson.

Belkaoui, A. \& Karpik, P. G. (1989). Determinants of the Corporate Decision to Disclose Social Information. Accounting, Auditing and Accountability Journal, 2(1), 36-51.https://doi.org/10.1108/09513578910132240

Broadbent, J. \& Laughlin, R. (1997). Development Empirical Research: An Example Informed by a Habermasian Approach. Accounting, Auditing and Accountability Journal, 10(5), 622-648. https://doi. org/10.1108/09513579710194027

Contabilidad y Negocios (15) 30, 2020 / ISSN 1992-1896 
Broadbent, J. \& Guthrie, J. (2008). Public Sector to Public Services: 20 years of "Contextual" Accounting Research. Accounting, Auditing \& Accountability Journal, 21(2), 129-169. https:/doi. org/10.1108/09513570810854383

Brown, J. \& Dillard, J. (2013). Agonizing over Engagement: SEA and the 'Death of Environmentalism' Debates". Critical Perspectives on Accounting, 24, 1-18. https://doi.org/10.1016/j.cpa.2012.09.001

Burrell, G. \& Morgan, G. (1979). Sociological Paradigm and Organizational Analysis. Londres: Heinemann.

Burritt, R. L., Hahn, T. \& Schaltegger, S. (2002). Towards a Comprehensive Framework for Environmental Management Accounting - Links between Business Actors and Environmental Management Accounting Tools. Australian Accounting Review, 12(2), 39-50. https:// doi.org/10.1111/j.1835-2561.2002.tb00202.x

Burritt, R. \& Schaltegger, S. (2001). Eco-efficiency in Corporate Budgeting. Environmental Management and Health, 12(2), 158-174. https://doi. org/10.1108/09566160110389924

Burritt, R. \& Schaltegger, S. (2010). Sustainability Accounting and Reporting: Fado r Trend? Accounting, Auditing and Accountability Journal, 23(7), 829-846. https://doi.org/10.1108/09513571011080144

Carrasco, F. \& Larrinaga, C. (1996). El poder constitutivo de la Contabilidad. Consideraciones sobre la cuestión medioambiental. En Ministerio de Economía, Industria y Competitividad \& Instituto de Contabilidad y Auditoría de Cuentas (eds.), Ensayos sobre Contabilidad y Economía. Homenaje al profesor Ángel Sáez Torrecilla (pp. 65-83). Madrid: Instituto de Contabilidad y Auditoría de Cuentas.

Chatfield, M. (1977). A History of Accounting Thought. Nueva York: Krieger.

Cho, C., Laine, M., Roberts, R. \& Rodrigue, M. (2015). Organized Hypocrisy, Organizational Façades, and Sustainability Reporting. Accounting, Organizations and Society, 40, 78-94. https://doi.org/10.1016/j. aos.2014.12.003

Cho, C. \& Patten, D. M. (2007). The Role of Environmental Disclosures as Tools of Legitimacy: A Research Note. Accounting, Organizations and Society, 32, 639647. https://doi.org/10.1016/j.aos.2006.09.009

Chua, W. F. (1986). Desarrollos radicales del pensamiento contable En M. Gómez-Villegas \& C. M. Ospina (2009). Avances interdisciplinarios para una comprensión critica de la contabilidad: Textos paradigmáticos de las corrientes heterodoxas. Bogotá: Universidad Nacional de Colombia - Universidad de Antioquia.

Cooper, C. \& Puxty, A. (1996). On the Proliferation of Accounting (His)tories. Critical Perspectives on Accounting, 7, 285-313. https://doi.org/10.1006/cpac.1996.0034

Cooper, D., Puxty, T., Lowe, T. \& Willmott, H. (1989) La profesión contable, el corporativismo y el Estado. En W. F. Chua, T. Lowe \& T. Puxty (eds.), Perspectivas criticas en control de gestión (pp. 245-270). Londres: Palgrave Macmillan. https://doi.org/10.1007/978-1349-07658-1_12

Deegan, C. (2002). Introduction. The Legitimizing Effect of Social and Environmental Disclosure - A Theoretical Foundation. Accounting, Auditing and Accountability Journal, 15(3). https://doi.org/10.1108/09513570210435852

Deegan, C. (2017). Twenty-five Years of Social and Environmental Accounting Research within Critical Perspectives on Accounting: Hits, Misses and Ways Forward. Critical Perspectives on Accounting, 43, 65-87. https://doi.org/10.1016/j.cpa.2016.06.005

Dierkes, M. \& Preston, L. (1977). Corporate Social Accounting Reporting for Physical Environment: A Critical Review and Implementation Proposal. Accounting, Organizations and Society, 2(1), 3-22. https://doi. org/10.1016/0361-3682(77)90003-4

Dillard, J. (1991). Accounting as Critical Social Science. Accounting, Auditing and Accountability Journal, 4(1). https://doi.org/10.1108/09513579110143849 
Dillard, J. (2014). Legitimating the Social Accounting Project: An Ethic of Accountability. Sustainability Accounting and Accountability. Londres: Routledge.

Epstein, M.J. (1996, Autumn). Improving Environmental Accounting with Full Environmental Cost Accounting. Environmental Quality Management, 6(1), 11-12. https://doi.org/10.1002/tqem.3310060104

Epstein, M., Flamholtz, E. \& McDonough, J. (1976). Social Corporate Accounting in United States. Accounting, Organizations and Society, 1(1), 23-42. https:// doi.org/10.1016/0361-3682(76)90005-2

Epstein, M. J. \& Freedman, M. (1994). Social Disclosure and the Individual Investor. Accounting, Auditing and Accountability Journal, 7(4), 94-109. https://doi. org/10.1108/09513579410069867

Evans, L. (2018). Language, Translation and Accounting: Towards a Critical Research Agenda. Accounting, Auditing \& Accountability Journal, 31(7), 1844-1873. https://doi.org/10.1108/AAAJ-08-2017-3055

Fassio, A. (2018). Reflexiones acerca de la metodología cualitativa para el estudio de las organizaciones. Ciencias Administrativas, 6(12), 73-84. https://doi. org/10.24215/23143738e 028

Feyerabend, P. K. (2003) Tratado contra el método. Madrid: Tecnos.

Foucault, M. (1987). Arqueología del saber. México, D. F.: Siglo XXI Editores.

Gadamer, H. G. (1992). Verdad y método. Salamanca: Sígueme.

Gaffikin, M. (2006). The Critique of Accounting Theory. Faculty of Business, Accounting \& Finance Working Papers 06/25. Wollongong: University of Wollongong.

Galeano, M. E. (2004). Diseño de proyectos de investigación cualitativa. Medellín: Fondo Editorial EAFIT.

Gil, J. M. (2018). Elogio de la contabilidad crítica. Teuken Bidikay, 9(12), 23-54. https://doi.org/10.33571/teuken.v9n12a1
Gómez, M. (2006). Una reflexión sobre la contabilidad como racionalidad instrumental en el capitalismo. Contaduria Universidad de Antioquia, 49, 87-94

Gómez, Y. \& Sarmiento, H. J. (2016). Elementos de un debate epistemológico por la nueva educación contable. Teuken Bidikay, 7(9). 55-73. Recuperado de https://revistas.elpoli.edu.co/index.php/teu/article/ view/1009/833 [Consulta: 14 de octubre de 2019]

Gracia, E. (2013). Habermas y su incidencia en la contabilidad. Lúmina, 14, 88-115. https://doi.org/10.30554/ lumina.14.1085.2013

Gray, R. (1992). Accounting and Environmentalism: An Exploration of the Challenge of Gently Accounting for Accountability, Transparency and Sustainability. Accounting Organizations and Society, 17(5), 399-425. https://doi.org/10.1016/0361-3682(92)90038-T

Gray, R. (2010). Is Accounting for Sustainability Actually Accounting for Sustainability... and How Would We Know? An Exploration of Narratives of Organizations and the Planet. Accounting, Organizations and Society, 35, 47-62. https://doi.org/10.1016/j. aos.2009.04.006

Gray, R., Adams, C. \& Owen, D. (2017). Social and Environmental Accounting. En R. Roslender (ed.), The Routledge Companion to Critical Accounting (pp. 243259). Londres: Routledge-Taylor \& Francis Group. https://doi.org/10.4324/9781315775203-14

Gray, R. \& Bebbington, J. (2001). Accounting for the Environment. Londres: SAGE.

Gray, R., Owen, D. \& Adams, C. (1996). Accounting \& Accountability: Changes and Challenges in Corporate Social and Environmental Reporting. Londres: Prentice Hall.

Guidry, R. \& Patten, D. (2012). Voluntary Disclosure Theory and Financial Control Variables: A Assessment of Recent Environmental Disclosure Research. Accounting Forum, 36(2), 81-90. https://doi.org/10.1016/j. accfor.2012.03.002 
Guthrie, J. \& Parker, L. D. (1990). Corporate Social Disclosure Practice: A Comparative International Analysis. Advances in Public Interest Accounting, 3, 159-175.

Habermas, J. (1988). La lógica de las ciencias sociales. Madrid: Tecnos.

Habermas, J. (1995). Conocimiento e interés. Valencia: Universitat de València.

Hopper, T., Lassou, P. \& Soobaroyen, T. (2017). Globalisation, Accounting and Developing Countries. Critical Perspectives on Accounting, 43, 125-148. https://doi. org/10.1016/j.cpa.2016.06.003

Hopwood, A. (1976). Accounting and Human Behavior. Englewood Cliffs: Prentice Hall.

Hopwood, A. (1983). On Trying to Study Accounting in the Contexts in Which it Operates. Accounting, Organizations and Society, 8(2-3), 287-305. https://doi. org/10.1016/0361-3682(83)90035-1

Hopwood, A. (2009). Accounting and Environment. Accounting, Organizations and Society, 34, 433-439. https://doi.org/10.1016/j.aos.2009.03.002

Khurana, I. K. \& Raman, K. K. (2004). Litigation Risk and the Financial Reporting Credibility of Big 4 versus Non-Big 4 Audits: Evidence from Anglo-American Countries. The Accounting Review, 79, (2), 473-495. https://doi.org/10.2308/accr.2004.79.2.473

Kuhn, T. S. (2006). La estructura de las revoluciones cientificas. México, D.F.: Fondo de Cultura Económica.

Laine, M. (2005). Meanings of the Term 'Sustainable Development' in Finnish Corporate Disclosures. Accounting Forum, 29, 395-413. https://doi.org/10.1016/j.accfor.2005.04.001

Lákatos, I. (1998). La metodología de los programas de investigación cientifica. Madrid: Alianza Editorial.

Larrinaga, C. (1997). Consideraciones en torno a la relación entre la contabilidad y el medio ambiente. Revista Española de Financiación y Contabilidad, 26(93), 957-991.
Larrinaga, C. (1999). Perspectivas alternativas de investigación en contabilidad: una revisión. Revista de Contabilidad, 2(3), 103-131.

Larrinaga, C. (2017). En torno a la evolución de la investigación en contabilidad social y medioambiental. Teuken Bidikay, 8(11), 21-38. https://doi.org/10.33571/teuken.v8n11a1

Larrinaga, C., Carrasco, F., Correa, C., Llena, F. \& Moneva. J. M. (2002). Accountability and Accounting Regulation: The Case of the Spanish Environmental Disclosure Standards. European Accounting Review, 11(4), 723-740. https://doi.org/10.1080/096381802 2000001000

Laudan, L. (1986). El progreso y sus problemas: Hacia una teoría del crecimiento cientifico. Madrid: Editorial Encuentro.

Lehman, C., Annisette, M. \& Agyemang, G. (2016). Immigration and Neoliberalism: Three Cases and Counter Accounts. Accounting, Auditing \& Accountability Journal, 29(1), 43-79. https://doi.org/10.1108/ AAAJ-09-2013-1470

Lehman, C. \& Ockabol, F. (2005). Accounting for Crime. Critical Perspectives on Accounting, 16, 613-639. https://doi.org/10.1016/j.cpa.2003.08.003

Lehman, C. \& Tinker, T. (1987). The 'Real' Cultural Significance of Accounts. Accounting, Organizations and Society, 12 (5), 503-522. https:// doi.org/10.1016/0361-3682(87)90033-X

Lehman, G. (2001). Reclaiming the Public Sphere: Problems and Prospects for Corporate Social and Environmental Accounting. Critical Perspectives on Accounting, 12 (6), 713-33. https://doi.org/10.1006/ cpac. 2001.0476

Lehman, G. (2006). Perspectives on Language, Accountability and Critical Accounting: An Interpretative Perspective. Critical Perspectives on Accounting, 17, 755-779. https://doi.org/10.1016/j.cpa.2005.02.002 
Lodh, S. \& Gaffikin, M. (1997). Critical Studies in Accounting Research, Rationality and Habermas: A Methodological Reflection. Critical erspectives on Accounting, 8 (5), 433-474. https://doi.org/10.1006/cpac.1996.0108

Lopes de Sá, A. (1995). Autonomía y calidad científica de la contabilidad. Revista de la Facultad de Contaduría Pública de la Universidad Autónoma de Bucaramanga, 17, 3-27.

Lowe, A. (2004). Postsocial Relations: Toward a Performative View of Accounting Knowledge. Accounting, Auditing \& Accountability Journal, 17(4), 604-628. https://doi.org/10.1108/09513570410554579

Lowe, T. \& Tinker, T. (1987). Accounting as Social Science: Abstract versus Concrete Sources of Accounting Change. En W. F. Chua, T. Lowe, T. Puxty (eds.), Critical Perspectives on Management Control (pp. 47-61). London: Palgrave MacMillan. https://doi. org/10.1007/978-1-349-07658-1_12

Martínez, V. \& Gómez-Villegas, M. (2015). La contabilidad y los conflictos ambientales en el sistema financiero: estudio de caso en el sector bancario argentino. Cuadernos de Contabilidad, 16(41). https://doi. org/10.11144/Javeriana.cc16-41.ccas

Mathews, R. (2004). Developing a Matrix Approach to Categorize the Social and Environmental Accounting Research Literature. Qualitative Research in Accounting \& Management, 1(1), 30-45. https://doi. org/10.1108/11766090410816280

Mathews, R. (2001). Some Thoughts on Social and Environmental Accounting Education. Accounting Education, 10(4), 335-352. https://doi.org/10.1080/ 09639280210121132

Mattessich, R. (2002). Contabilidad y métodos analíticos. Buenos Aires: La Ley.

Maunders, K. \& Burritt, R. L. (1991). Accounting and Ecological Crisis. Accounting, Auditing and Accountability Journal, 4(3). https://doi.org/10.1108/0951357 9110003277
Mc Phail, K., Bebbington, J. \& Gray, R. (1994). Teaching Ethics in Accounting and the Ethics of Accounting Teaching: Educating for Immorality and a Possible Case for Social and Environmental Accounting Education. Accounting Education, 3(1). 51-75. https://doi. org/10.1080/09639289400000005

Mirvis, P. H. \& Lawler, E. E. (1984). Accounting for the Quality of Work Life. Journal of Organization Behavior, 5(3), 197-212. https://doi.org/10.1002/ job.4030050304

Moneva, J. M., Archel, P. \& Correa, C. (2006). GRI and the Camouflaging of Corporate Unsustainability. Accounting Forum, 30, 121-137. https://doi.org/10.1016/j. accfor.2006.02.001

O’Dwyer, B. \& Owen, D. (2005). Assurance Statement Practice in Environmental, Social and Sustainability Reporting: A Critical Evaluation. The British Accounting Review, 37, 205-229. https://doi.org/10.1016/j. bar.2005.01.005

O’Dwyer, B., Owen, D. \& Unerman, J. (2011). Seeking Legitimacy for New Assurance Forms: The Case of Assurance of Sustainability Reporting. Accounting, Organizations and Society, 36, 31-52. https://doi. org/10.1016/j.aos.2011.01.002

Owen, D. (2008). Chronicles of Wasted Time? A Personal Reflection on the Current State of, and Future Prospects for, Social and Environmental Accounting Research. Accounting, Auditing \& Accountability Journal, 21(2), 240-267. https://doi. org/10.1108/09513570810854428

Owen, D. L., Swift, T. A., Humphrey, C. \& Bowerman, M. (2000). The New Social Audits: Accountability, Managerial Capture or the Agenda of Social Champions? European Accounting Review, 9(1), 81-99. https://doi.org/10.1080/096381800407950

Parker, L. D. (1987). The Impact of Corporate Characteristics on Social Responsibility Disclosure: A Typology and Frequency-Based Analysis. Accounting,

Contabilidad y Negocios (15) 30, 2020 / ISSN 1992-1896 
Organizations and Society, 12(2), 111-122. https://doi. org/10.1016/0361-3682(87)90001-8

Parker, L. D. (2005). Social and Environmental Accountability Research: A View from the Complementary Box. Accounting, Auditing \& Accountability Journal, 18(6), 842-860, https://doi.org/10.1108/09513570 510627739

Peloza, J. (2009). The Challenge of Measuring Financial Impacts from Investment in Corporate Social Performance. Journal of Management, 35(6), 1518-1541. https://doi.org/10.1177/0149206309335188

Peloza, J. (2011). How Can Corporate Social Responsibility Activities Create Value for Stakeholders? A Systematic Review. Journal of the Academy of Marketing Science, 39(1), 117-135. https://doi.org/10.1007/ s11747-010-0213-6

Piketty, T. (2014). El capital en el siglo XXI. México, D. F.: Fondo de Cultura Económica.

Popper, K. R. (2008). La lógica de la investigación cientifica. Madrid: Tecnos.

Preston, L. E. (1978). Analyzing Corporate Social Performance: Methods and Results. Journal of Contemporary Business, 7, 135-150. https://doi.org/10.1007/978-1349-05944-7_14

Preston, L. E. (1981). Research on Social Corporate Reporting. Directions for Development. Accounting, Organizations and Society, 6(3), 255-262. https://doi. org/10.1016/0361-3682(81)90031-3

Preston, L., O’Bannon, E. \& Douglas P. (1997). The Corporate Socio-Financial Performance Relationship. Business and Society, 36, 419-429. https://doi. org/10.1177/000765039703600406

Puxty, A., Willmott, H., Cooper, D. \& Lowe, T. (1987). Modes of Regulation in Advance Capitalism: Locating Accountancy in Four Countries. Accounting, Organizations and Society, 12(3), 273-291. https://doi. org/10.1016/0361-3682(87)90041-9
Qiu, Y., Shaukat, A. \& Tharyan, R. (2016). Environmental and Social Disclosures: Link with Corporate Financial Performance. The British Accounting Review, 48, 102116. https://doi.org/10.1016/j.bar.2014.10.007

Richardson, A. J. (1987). Accounting as Legitimating Institution. Accounting, Organizations and Society, 12(4), 341-355. https://doi.org/10.1016/0361-3682 (87)90023-7

Schmandt-Besserat, D. (1999). The History of Counting. Nueva York: Harper-Collins

Shaukat, A., Qiu, Y. \& Trojanowsky, G. (2016). Board Attributes, Corporate Social Responsibility Strategy, and Corporate Environmental and Social Performance. Journal of Business Ethics, 135(3), 569-585. https:// doi.org/10.1007/s10551-014-2460-9

Sikka, P., Willmott, H. \& Lowe, T. (1989). Guardians of Knowledge and Public Interest: Evidence and Issues of Accountability in the UK Accountancy Profession. Accounting, Auditing \& Accountability Journal, 2(2). https://doi.org/10.1108/09513578910132286

Solomon, J. F. \& Solomon, A. (2006). Private Social, Ethical and Environmental Disclosure. Accounting, Auditing \& Accountability Journal, 19(4), 564-591. https://doi.org/10.1108/09513570610679137

Spence, C. (2007). Social and Environmental Reporting and Hegemonic Discourse. Accounting, Auditing \& Accountability Journal, 20(6), 855-882. https://doi. org/10.1108/09513570710830272

Spence, C. (2009). Social Accounting's Emancipatory Potential: A Gramscian Critique. Critical Perspectives on Accounting, 20, 205-227. https://doi.org/10.1016/ j.cpa.2007.06.003

Spence, C., Husillos, J. \& Correa-Ruiz, C. (2010). Cargo Cult Science and the Death of Politics: A Critical Review of Social and Environmental Accounting Research. Critical Perspectives on Accounting, 21, 76-89. https://doi.org/10.1016/j.cpa.2008.09.008 
Thomson, I. (2015). But Does Sustainability Need Capitalism or an Integrated Reporting? A Commentary on "The International Integrated Reporting Council, a Story of Failure" by Flower J. Critical Perspectives on Accounting, 27, 18-22. https://doi.org/10.1016/j. cpa.2014.07.003 Tinker, T. \& Gray, R. (2003). Beyond a Critique of Pure Reason: From Policy to Politics to Praxis in Environmental and Social Research. Accounting, Auditing \& Accountability Journal, 16(5), 727-761. https://doi.org/10.1108/09513570310505952

Tinker, T., Neimark, M. \& Lehman, C. (1991). Falling down the Hole in the middle of the Road: Political Quietism in Corporate Social Reporting. Accounting, Auditing \& Accountability Journal, 4(2). https://doi. org/10.1108/09513579110000504

Ullmann, A. (1976). The Corporate Environmental Accounting System: A Management Tool for Fighting Environmental Degradation. Accounting, Organization and Society, 1(1), 71-79. https://doi.org/10.1016/ 0361-3682(76)90008-8

Unerman, J. \& O'Dwyer B. (2006). Theorising Accountability for NGO Advocacy. Accounting, Auditing \& Accountability Journal, 19(3). 349-376. https://doi. org/10.1108/09513570610670334
Vasco, C.E. (1990). Tres estilos de trabajo en ciencias sociales. Comentarios a propósito del artículo Conocimiento e Interés de Jürgen Habermas. Bogotá: Centro de Investigación y Educación Popular (CINEP).

Wahyudi, I. (1999). Mainstream Accounting and its Paradigm: A Critical Analysis. International Journal of Business, 1(2), 99-112, https://doi.org/10.22146/ gamaijb.37907

Weber, M. (2003). La ética protestante y el espiritu del capitalismo. México, D.F.: Fondo de Cultura Económica.

Yongvanich, K. \& Guthrie, J. (2006). An Extended Performance Reporting Framework for Social and Environmental Accounting. Business Strategy and the Environment, 15, 309-321. https://doi.org/10.1002/ bse. 541

Zemelman, H. (2004). Pensar teórico y pensar epistémico. En I. Sánchez \& R. Sosa (coords.), América Latina: los desafíos del pensamiento crítico (pp. 21-33). México, D. F.: Siglo XXI.

Fecha de recepción: 15 de julio de 2020 Fecha de aceptación: 23 de octubre de 2020 Correspondencia: hjsarmiento63@gmail.com 